\title{
MODELOS DE INTERACCIÓN DE LOS ADOLESCENTES EN CONTEXTOS PRESENCIALES Y VIRTUALES
}

\section{MODELS OF ADOLESCENT INTERACTION IN FACE AND ON-LINE ENVIRONMENTS}

\author{
Må del Mar Sánchez Vera; mmarsanchez@um.es \\ Ma Paz Prendes Espinosa; pazprend@um.es \\ José Luis Serrano Sánchez; jl.serranosanchez@um.es \\ Facultad de Educación. Universidad de Murcia
}

\section{RESUMEN}

Nativos digitales, generación-red o cibergeneración. Cualquiera de estos términos hace referencia a una generación de personas que han crecido con las TIC como parte de su entorno natural y esta generación es la que ocupa nuestras aulas. Por otra parte, son nuestros hábitos de comunicación uno de los aspectos de nuestras vidas que más ha cambiado por la influencia de las TIC. En este contexto se sitúa el interés del proyecto CIPRECES financiado por el MEC: conocer los hábitos de comunicación social de los estudiantes de Enseñanza Secundaria. En el marco de este proyecto hemos de entender este artículo que presenta una parte del mismo, concretamente hemos intentado describir los intercambios comunicativos de los adolescentes utilizando que modelos de interacción a través del análisis de sociogramas que nos permiten además establecer comparaciones entre sus relaciones presenciales y sus relaciones en el espacio virtual.

PALABRAS CLAVE: Comunicación presencial, comunicación virtual, interacción, relaciones sociales, adolescencia, enseñanza secundaria.

\section{ABSTRACT}

Digital natives, net generation or cibergeneration. Each of these terms refers to people that have grown up with ICT as natural environment and actually they are the students in our classrooms. By other hand, the most important change in our lives caused by ICT is communication and social interaction. In this context we have to put our project CIPRECES founded by spanish Ministry of Education with the aim to know about social communication habits of secondary students. Inside this project we have developed some interaction models using sociograms that describe social relations between teenagers both in presencial 
and in virtual contexts. Finally these models are useful to compare these types of interactions: face to face and virtual.

KEYWORDS: Face to face communication, virtual communication, interaction, social relations, teenagers, secondary school.

\section{INTRODUCCIÓN}

\section{Las redes como factor de aislamiento o como elemento de socialización}

Durante la década de los 90, cuando se produjo un incremento considerable de usuarios de Internet, los estudiosos vaticinaban que se produciría un aislamiento social del individuo. Como ejemplo de ello, un estudio publicado en 1998 en la revista American Psychologist concluía que el uso de Internet disminuía las relaciones sociales. Por su parte Castells, al contrario de Mcluhan cuando predecía la aldea global, señalaba que a consecuencia del uso de Internet acabaríamos siendo "chalecitos individuales, producidos a escala global y distribuidos localmente" (1996: 373).

Pero el transcurrir de los años no les ha dado la razón, sino más bien lo contrario. Matute (2002) criticó las conclusiones del mencionado estudio de 1998, poniendo en duda la adecuación de su metodología y aludiendo a que las investigaciones actuales muestran resultados totalmente contrarios. Igualmente el trabajo de Kraut, quien en la década de los 90 declaró haber encontrado que Internet favorecía el aislamiento social (1998), por contra posteriormente en un estudio realizado en el 2001 declara que los resultados obtenidos en el 98 habían desaparecido prácticamente, y que esto era debido a que Internet se había transformado en esos tres años (citado por Herrero, Meneses, Valiente y Rodríguez, 2004).

En este sentido es importante considerar el cambio comunicativo que ha llegado aparejado a la denominada "web 2.0" que ofrece al internauta la oportunidad de participar, publicar, compartir,... así como las diversas herramientas telemáticas que posibilitan la creación y el desarrollo de redes sociales y de todo tipo de espacios virtuales de comunicación e intercambio.

Nadie puede cuestionar la influencia de la Web 2.0 en los nuevos modelos de interacción social, puesto que ésta permite y potencia espacios virtuales para la participación y la interacción social gratuita basada en aplicaciones telemáticas intuitivas y fáciles de manejar, siendo "una revolución social que busca una arquitectura de la participación a través de aplicaciones y servicios abiertos" (Castaño, 2007, p. 240). Coincidiendo con Domínguez y Llorente $(2009$, p. 108) "la web 2.0 potencia espacios de interacción social, la participación colectiva y gratuita basada en tecnologías abiertas, flexibles y fáciles de utilizar por los internautas".

De este modo, encontramos en esta última década una serie de estudios que declaran que el uso de las redes favorece las relaciones sociales (Wellman, 2001; Herrero, Meneses, Valiente y Rodríguez, 2004; DiMaggio et al., 2001; Müller, 1999; Pérez y Gutiérrez, 2007; Aranda et al., 2010).

Herrero y cols. (2004) realizan un estudio con alumnos de psicología de edades comprendidas entre 19 y 57 años y el resultado que obtienen es que ambas formas de 
participación (virtual y presencial) se relacionan positivamente, con influencia positiva en la autoestima social, y ambas favorecen el mantenimiento de vínculos sociales satisfactorios.

Por su parte Aranda et al. (2010) concluyen que los jóvenes españoles entre 12 y 18 años hacen una "extensión online de la vida offline" debido a su alto nivel de integración en su vida cotidiana, siendo estas "herramientas esenciales en relación con la sociabilidad de los y las adolescentes". Este estudio revela además que los contactos realizados estrictamente online se reducen a porcentajes mínimos.

El estudio Pew Internet Project elaborado en 2006 por un grupo de sociólogos canadienses de la Universidad de Toronto igualmente corroboró que las tecnologías de la información y las comunicaciones, como el correo electrónico, la mensajería instantánea y los móviles, lejos de aislar a las personas en el ciberespacio, fomentan las relaciones sociales y potencian la integración de los individuos en la vida cotidiana. Se señala además que internet no hace que las otras relaciones sociales de proximidad (como por ejemplo las familiares) vayan en detrimento; el norteamericano medio tiene unas 35 relaciones estrechas con personas, mientras que en el caso de los internautas esta media se enumera en 37.

\section{La comunicación a través de herramientas télemáticas}

El elemento clave que caracteriza el impacto social de las Nuevas Tecnologías es que la "comunicación ha roto sus fronteras" (Prendes, 2005: 131), siendo principalmente los menores de 30 años quienes más facilidades tienen en el momento de incorporar cualquier nueva tecnología así como nuevas modalidades de comunicación (Prendes, 2005). En esta misma línea de pensamiento, Martínez y Solano (2003: 15) opinan que dichas redes telemáticas han influido en la forma de "acceder a la información y de materializar ésta en conocimiento, así como en la delimitación de pautas de comportamiento social, no sólo en el espacio de las redes (espacios virtuales) sino también en el contexto presencial de la comunicación".

Según Martínez (2007, p. 41) los modelos de comunicación en la nueva situación tecnológica son "situaciones de comunicación dilatadas en el espacio si bien puede hablarse de situaciones presenciales aceptando la creación de un espacio virtual de comunicación en el que se desarrolla el proceso y que los interlocutores comparten". Rizo $(2006, p .6)$, considera que con el auge de las TIC "vivimos una suerte de revolución sociocultural que permite la creación y consolidación de nuevas modalidades de comunicación $y$, por tanto, de interacción", siendo esta una de las dimensiones más importantes de la comunicación. Desde el ámbito educativo, se considera la interacción como uno de los elementos esenciales del proceso educativo (concepción constructivista), pudiendo definir este elemento como una acción recíproca entre dos más agentes siendo interesante destacar que el resultado es siempre la modificación de los estados de los participantes (Rizo, 2006).

La comunicación en la red y la organización social es tema de interés de varios grupos de investigación europeos y nacionales, así por ejemplo el Grupo de investigación, Orientación y Desarrollo Educativo (GRODE) de la Universidad Autónoma de Barcelona lanzó en 2005 el Proyecto Software Sociograma (http://sociograma.grode.org/inicio.aspx), una herramienta para intervenir en la dinámica del aula y analizar las relaciones sociales. La AECT (Asociación para Comunicaciones Educativas y Tecnología) (http://www.aect.org/About/History/\#impact) es una organización europea que tiene como uno de sus objetivos el de definir las disciplinas y las actividades profesionales que comprenden el campo de comunicaciones educativas y tecnología. 
Coincidiendo con Moral (2009) y Meneses (2006), existe un continuo crecimiento de los servicios con finalidad personal o social debido a la implantación de las TIC, concretamente de Internet, siendo un espacio de interacción social. Sin duda, ha de tenerse muy en cuenta las posibilidades que las diversas herramientas y entornos tecnológicos ofrecen actualmente como valiosos instrumentos para fomentar la interacción.

Para Moral (2009, pp. 232-233) la comunicación y la interacción electrónica pueden traer una serie de consecuencias negativas y positivas. Respecto a las primeras: "mayor dificultad de llegar a acuerdos en grupo y la presentación frecuente de comportamientos hostiles y agresivos; relaciones poco profundas e impersonales; un menor grado de autoconciencia e identidad individual; una disminución de la participación social y del bienestar psicológico; un mayor grado de falsedad en los datos personales de los participantes". Respecto a las consecuencias positivas serían: "que en la interacción a través del ordenador, los usuarios habituales pueden conseguir una comunicación con un contenido socioemocional igual o superior al que puedan darse en las interacciones cara a cara; la consecuencia de un nivel de intimidad muy alto en periodos muy breves de tiempo; la igualación del estatus de los participantes; la posibilidad de poner de manifiesto aspectos ocultos de la personalidad y de incrementar la autoestima, el incremento del círculo social y la incorporación de las relaciones personales del espacio virtual al espacio real".

\section{La generación red}

Se da además la situación de que los alumnos que se encuentran en nuestro sistema escolar pertenecen a la llamada net-generation, una generación que inicia su existencia con recursos o medios para comunicarse de manera electrónica a través de una red (Edel, 2006). Según el informe "Infancia y Adolescencia en la Sociedad de la Información" del Observatorio de las Telecomunicaciones de Red.es (2005) el 63\% de los hogares con niños de hasta 15 años está conectado a Internet, además el $66 \%$ de los niños y el $84 \%$ de los jóvenes son internautas y ambos grupos usan Internet con más frecuencia que los adultos. Es decir, nos encontramos ante unos alumnos que son usuarios formales de los ordenadores, que trabajan con las TIC (Tecnologías de la Información y la Comunicación) en la escuela, en el hogar, y las utilizan como fuente de entretenimiento y medio de comunicación.

Muchos foros de discusión sobre TIC proponen olvidar el concepto de nativos e inmigrantes digitales de Prensky (2001) y lo redefinen en torno a los conceptos de visitantes y residentes. De acuerdo con las ideas de White (2010), un residente digital es alguien que incluye parte de su identidad en Internet, que utiliza la tecnología como algo habitual y que interacciona con otras personas a través de las redes, un residente pasa un porcentaje significativo de su vida en red. Un visitante, por contra, haría referencia a las personas que utilizan la tecnología para actividades concretas y relativamente esporádicas, como reservar las vacaciones mediante Internet. Los visitantes pueden pasar un determinado periodo de tiempo en la red pero no "residen" en ella, es decir, no sienten que tienen la necesidad de participar en Internet de la misma manera que lo tendría un residente. Podríamos pensar que muchos de los considerados "nativos digitales" que Prensky describía en 2001 como aquellos que no habían cumplido los 30 años y que pasaban gran cantidad de horas en Internet desarrollando tareas de su vida cotidiana, pueden formar parte del grupo denominado "residentes virtuales", que evidentemente es mucho más amplio que el de nativos digitales, ya que no tiene la connotación de la edad. Sea como fuere, lo que parece claro es que los jóvenes utilizan las TIC mucho, forman parte de su entorno natural y proporcionan la posibilidad de desarrollar nuevos mecanismos para comunicarse. Los últimos estudios avalan esta idea. 
El sexto observatorio de tendencias de la empresa Nokia afirma que el 53\% de los jóvenes españoles entre 15 y 35 años tienen Internet en el teléfono móvil. Un estudio financiado en realizado por Del Río, Sábada y Bringué (2010) indica que a los 12 años prácticamente la mitad de jóvenes españoles (un 44,9\%) tiene ya Tuenti y lo usa con asiduidad. Y conforme aumenta paulatinamente la edad este porcentaje aumenta del tal modo que a los 16 años más del $90 \%$ de los jóvenes se encuentra ya en la nombrada red social. De acuerdo con las ideas que expone Mir (2010), lo importante es reconocer que los jóvenes participan mucho en la red, no cuestionamos si son competentes digitalmente o no, pero sí es evidente que los adolescentes emplean una gran parte de su tiempo en un entorno digital.

Es por tanto de gran interés el estudio y análisis de las relaciones sociales que se establecen en el ciberespacio y más aún si se pueden analizar los organigramas que se desprenden de las relaciones en ambos contextos, presencial y mediado tecnológicamente. Tal es el contexto de nuestra investigación, el proyecto "CIPRECES (Hábitos de Comunicación y Relaciones Sociales de los estudiantes en entornos Presenciales y Virtuales)", un proyecto de I+D financiado por el Ministerio de Ciencia e Innovación dentro del VI Plan Nacional de Investigación y Desarrollo e Innovación Tecnológica. Ha sido dirigido por el Dr. Francisco Martínez de la Universidad de Murcia y el grupo que firma el artículo ha sido el encargado del análisis de los modelos de interacción que de forma sumaria presentamos. El proyecto se ha desarrollado desde 2008 a 2011 y ha tenido como finalidad la de conocer los hábitos de comunicación social de los estudiantes en edad de escolarización secundaria para describir los intercambios comunicativos y la relación entre los modelos de interacción a través del análisis de sociogramas.

\section{DESCRIPCIÓN DE LA METODOLOGÍA}

El trabajo de investigación ha definido como población la de los alumnos de segundo ciclo de Enseñanza Secundaria Obligatoria principalmente por dos aspectos prioritarios: en primer lugar, porque estos cursos son los finales de la etapa de educación obligatoria, por lo que tienen una formación focalizada en la continuación de sus estudios hacia un nivel de mayor profesionalización o bien a la incorporación posterior al mundo del trabajo; y en segundo lugar, porque son alumnos nacidos con posterioridad a 1994, luego pueden ser considerados generacionalmente como usuarios activos de las TIC -tal y como se ha podido conocer en los estudios reflejados anteriormente- en una época en la que España ya está integrada en las tecnologías móviles y la Web.

El proyecto se ha centrado en dos líneas de trabajo principales. En la primera se ha realizado una descripción de los hábitos de comunicación -presenciales y en red- de los alumnos participantes, analizando también el uso de diferentes herramientas de comunicación social, para lo cual el instrumento de recogida de información que hemos utilizado ha sido un cuestionario. Tras los datos obtenidos en esa primera fase, hemos llevado a cabo la segunda parte del estudio: hacer un análisis pormenorizado de los mapas o modelos de interacción que caracterizan las relaciones sociales de los alumnos de secundaria en los diferentes contextos (presencial y virtual). Esta segunda fase de trabajo es la que se presenta en el este artículo. 


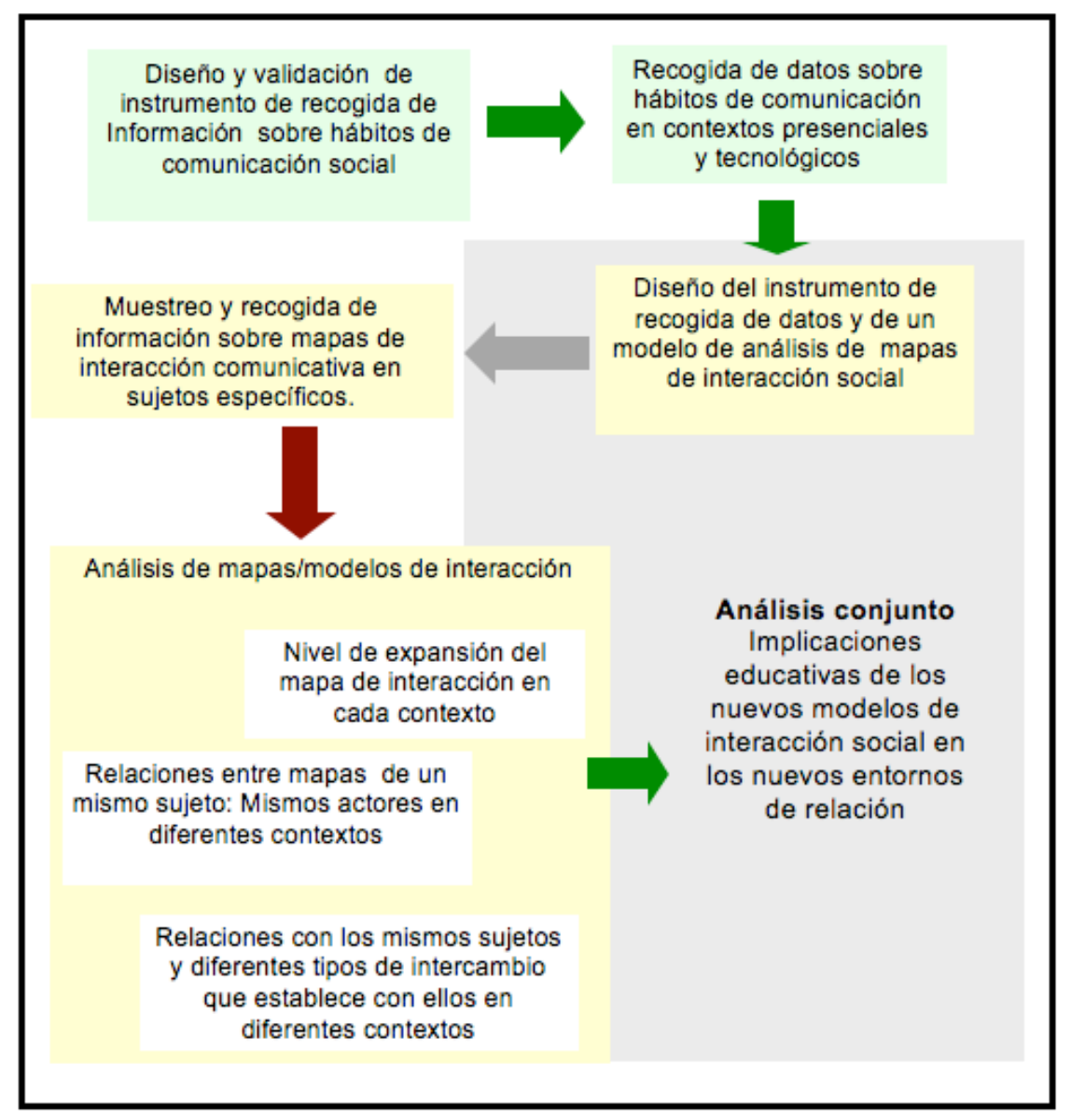

Figura 1. Procedimiento de análisis de los modelos de interacción.

La población de sujetos de nuestro estudio incluye a los alumnos del segundo ciclo de la enseñanza secundaria obligatoria (de 14 a 16 años) del territorio nacional. El método de muestreo utilizado ha sido no probabilístico y la técnica de selección de la muestra ha sido el muestreo por cuotas, ya que la selección se ha realizado entre los centros que reúnen unas determinadas condiciones o criterios de selección. De acuerdo con esta técnica, se ha seleccionado una muestra de Institutos de Educación Secundaria (IES) de siete provincias españolas (Alicante, Huelva, Las Palmas de Gran Canaria, Málaga, Oviedo, Palma de Mallorca y Murcia), coincidiendo éstas con la presencia de miembros del equipo de la investigación en ellas.

Para la realización de los mapas de interacción de los alumnos se partió de técnicas utilizadas habitualmente en sociometría que proporcionan la posibilidad de evaluar en poco tiempo y con gran validez las relaciones entre sujetos, permitiendo obtener información tanto de las relaciones establecidas por el alumnos, como de los contextos en los que se desarrolla. Dichas técnicas consisten en preguntar a todos los miembros de un determinado grupo (por ejemplo de una clase) acerca del resto y conocer así el estatus medio o nivel de popularidad, las oportunidades para el establecimiento de relaciones de amistad y los atributos perceptivos por los que más destaca.

Una vez clarificadas las variables que se debían tener en cuenta en función de los objetivos que se trazaron, se precisaron las herramientas de comunicación telemática que más interesaba estudiar en el contexto virtual para poder preguntar por las relaciones del alumno en cada una de ellas y de ese modo se construyó un instrumento de recogida de datos en el que se pidió al alumno nombrar a los sujetos que formaran parte de sus redes 
de comunicación en entornos tecnológicos y presenciales y que tipificaran qué tipo de relación establecían con ellos prioritariamente en cada uno de los contextos (personal, social y académico).

Así pues, dado el tipo de diagrama que se pretendía y el nivel de estudio de las relaciones que se realizaron, se utilizó una técnica de recogida de datos de pregunta directa. En todos los casos se les preguntaba su preferencia respecto a 1) con quién harían los deberes, 2) quedarían para divertirse o 3) hablarían de su vida personal y/o problemas. Además se pedía esta enumeración de forma específica en relación con otros tres aspectos: 1) si quedarían con ellos en su vida cotidiana de forma presencial, 2) con las que contactarían utilizando el móvil, y 3) las cinco personas con las que harían tales actividades a través de Internet (en este caso se citaban Tuenti, Facebook y Messenger, herramientas que el estudio previo reveló que eran las más utilizadas).

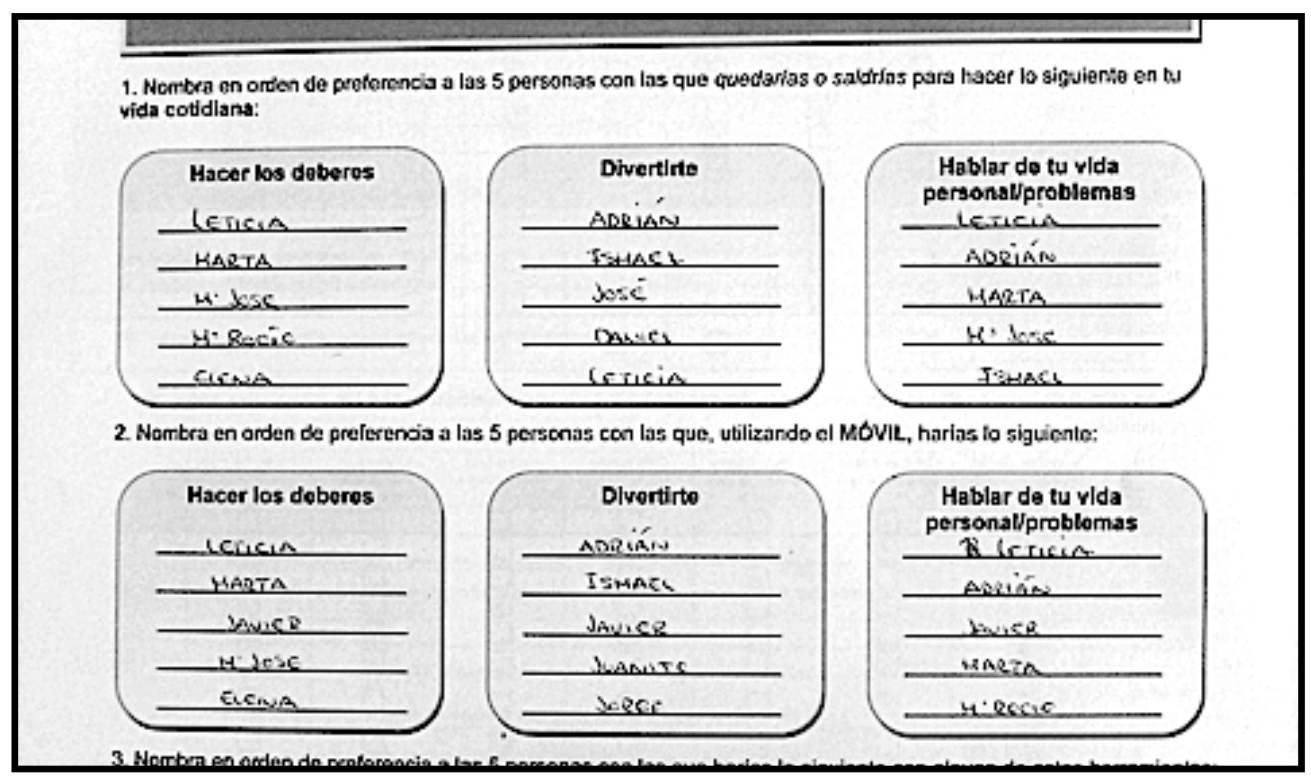

Figura 2: ejemplo de un cuestionario. Indicar las 5 personas con las que se realizarían actividades en la vida cotidiana y a través del móvil 


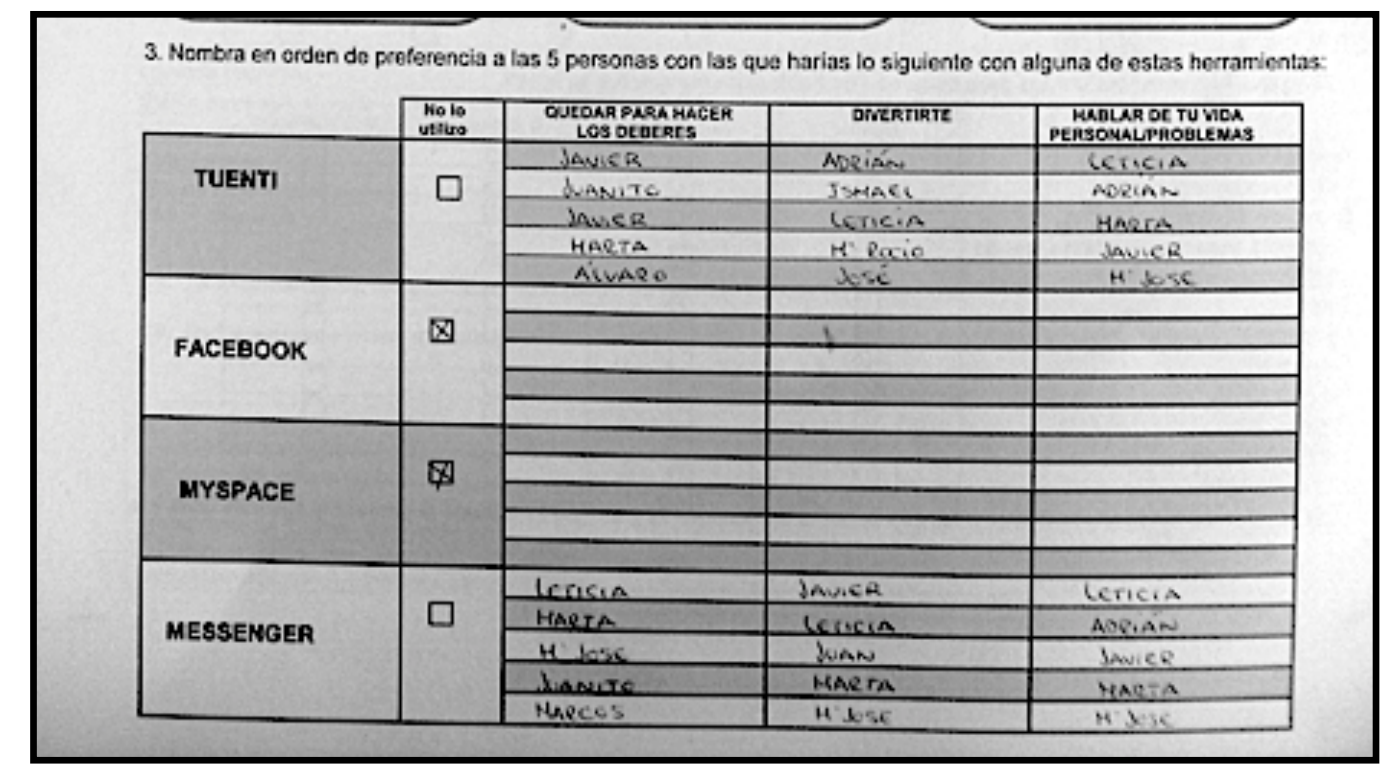

Figura 3: ejemplo de un cuestionario. Indicar las 5 personas con las que se realizarían actividades en las diferentes herramientas de la red

Posteriormente estos nombres se codificaron en números y se procedió a conocer cuántas veces aparecía ese contacto en cada una de las preguntas. Es decir, como podemos ver en la figura 2, Leticia es la primera persona que el encuestado incluye y afirma que es la persona con la que hace los deberes, esa misma Leticia aparece (todavía en el entorno presencial) como una persona con la que queda para actividades de ocio, aunque aparece en último lugar (no es la favorita). Leticia aparece de nuevo en el entorno presencial en primer lugar como la persona con la que más confianza tiene para comentar asuntos personales. Si nos vamos a las preferencias mediante móvil Leticia aparece en primer lugar para hacer los deberes y para tratar temas personales pero ya no aparece como una persona con la que habla para divertirse. Lo mismo sucede en los contactos indicados en las otras herramientas por las que se les preguntaba (Facebook, Tuenti, Messenger). Asignándole a Leticia el código numérico 1 , se puede comprobar cuántas veces aparece y dónde e igualmente con el resto de contactos. De este modo se pueden encontrar contactos con los que únicamente el sujeto se relaciona presencialmente, contactos con los que únicamente se relaciona tecnológicamente y/o en red y contactos que aparecen en ambos entornos.

\section{RESULTADOS ALCANZADOS}

Una vez obtenidos los datos de todos los alumnos se pudieron observar una serie de rasgos comunes que permitían agrupar a los alumnos para observar distintas tendencias y modelos de interacción. Básicamente, para la elaboración de los mapas de interacción se ha tenido en cuenta la manera en la que se relacionan los contactos del entorno tecnológico y del entorno presencial. Tras la observación de los resultados hemos definido cuatro modelos de interacción.

a) Modelo de costumbres: identificación de lo presencial y lo tecnológico.

El primer mapa de interacción (figura 3) corresponde al 27,11\% de los sujetos de la muestra, lo cual pone de manifiesto que un número considerable de sujetos se relacionan con las mismas personas en contextos presenciales y virtuales. 


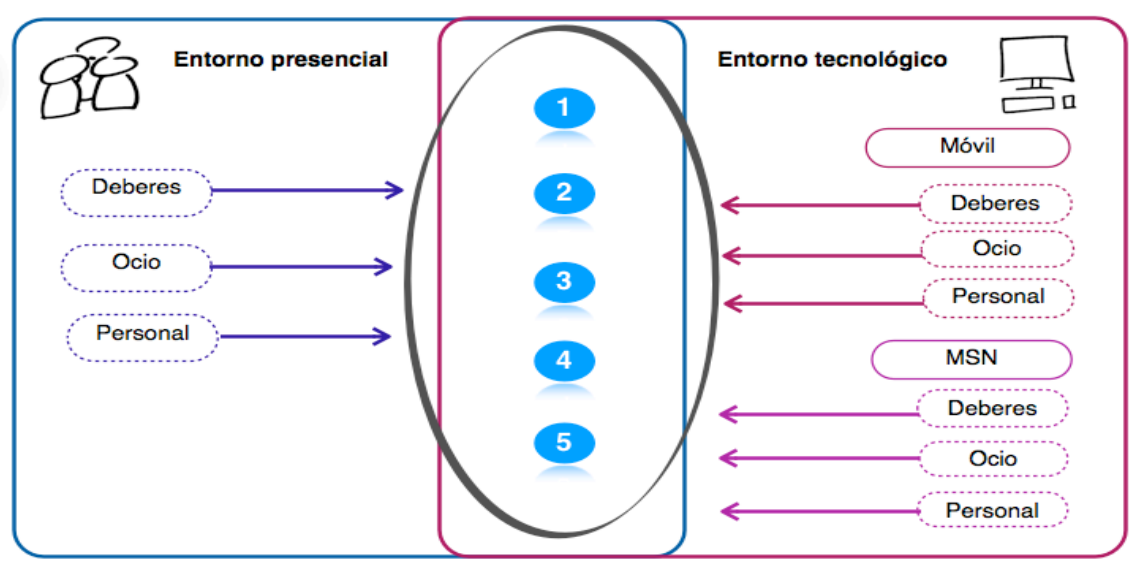

Figura 4. Ejemplo de mapa de interacción del "modelo de costumbres".

Las características básicas de los alumnos ubicados en este grupo son las siguientes: el estudiante cuenta siempre con la misma red de contactos para interactuar en el contexto presencial, con el móvil y en Internet. Se puede afirmar que utiliza el entorno tecnológico como complemento a la comunicación en su entorno social inmediato y presencial, pero no varía en sus interacciones (no incluye nuevos contactos). En estos casos es frecuente además que coincidan las personas con los que realiza cada tipo de actividad (educativa, de ocio o personal), identificándose por tanto que la elección que realiza de la persona que "estudia" en su entorno presencial es también con la que ejerce esas tareas en la red o a través del móvil. Se observa que estos sujetos son los que más actividades extraescolares realizan, como por ejemplo, pertenecer a un grupo scout, lo cual indica que su entramado social presencial es más amplio que el de los sujetos que encontramos en otros modelos. Quizás por ello también se destaca que los sujetos utilizan menos herramientas tecnológicas que en los otros modelos resultantes de nuestro estudio. Se utiliza principalmente el messenger en detrimento de las herramientas de red social (Tuenti y Facebook) que parecen no interesar a estos sujetos.

\section{b) Modelo de costumbres tecnológicamente ampliado.}

El segundo agrupamiento de alumnos (figura 4) se puede entender como una evolución del primer agrupamiento y con él se identifican el 53,2\% de los alumnos estudiados. La diferencia con el modelo anterior es que existen contactos nuevos con los que se comunica únicamente a través de Internet y que no aparecen en su contexto real. Una de las diferencias principales respecto al primer agrupamiento es que, además, las relaciones se diversifican, es decir, en el caso anterior un mismo contacto es identificado para diversas actividades como estudiar, ocio o personal, mientras que por el contrario en este modelo aparecen distintos contactos según la actividad. En este modelo los sujetos identifican como máximo 15 contactos, pues los casos donde es mayor el número de contactos se han incluido en otro de los modelos. 


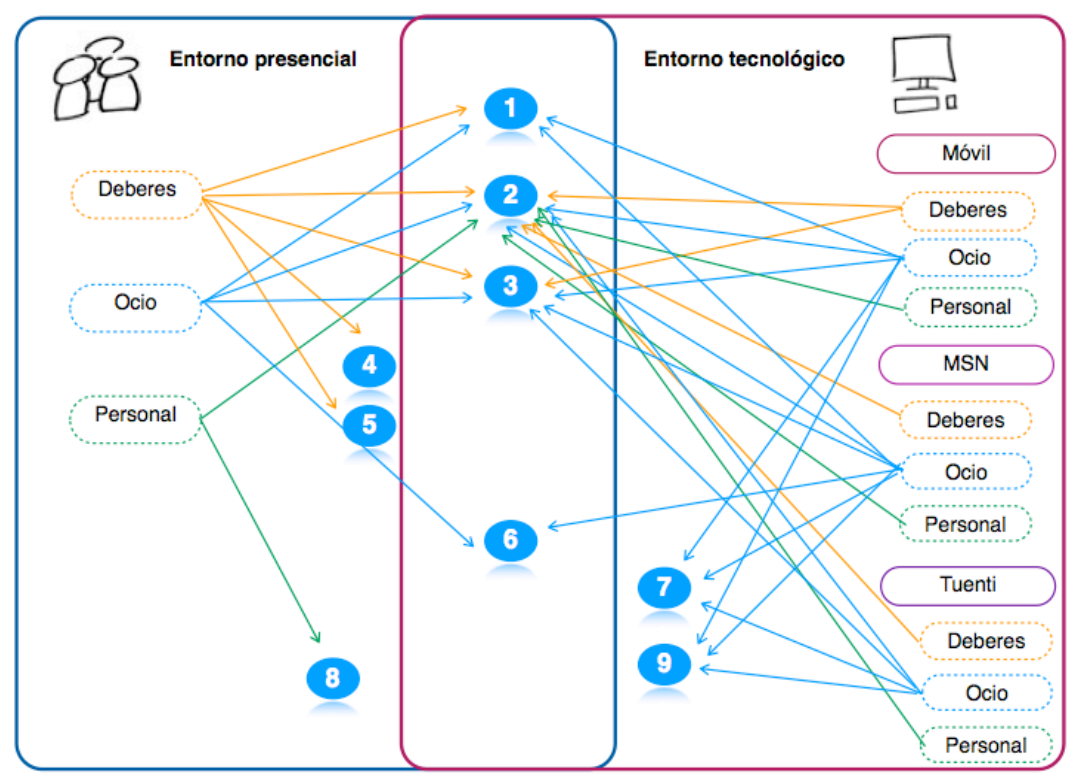

Figura 5. Ejemplo de mapa de interacción del "modelo tecnológicamente ampliado".

\section{c) Modelo tecnológico}

El tercer agrupamiento de sujetos (figura 5) se corresponde con el 10,45\% de la muestra. En este caso encontramos a los estudiantes que desarrollan un gran número de contactos únicamente en la red o son contactos con los que tienen poca relación en la presencialidad. En su mayoría este grupo de alumnos utiliza Tuenti más que Messenger, rompiendo con la tendencia encontrada hasta ahora en el resto de agrupamientos. Además, gracias al cuestionario se puede saber que estos alumnos utilizan la red principalmente para llevar a cabo actividades de ocio y de interrelación personal, sobre todo para conocer a personas.

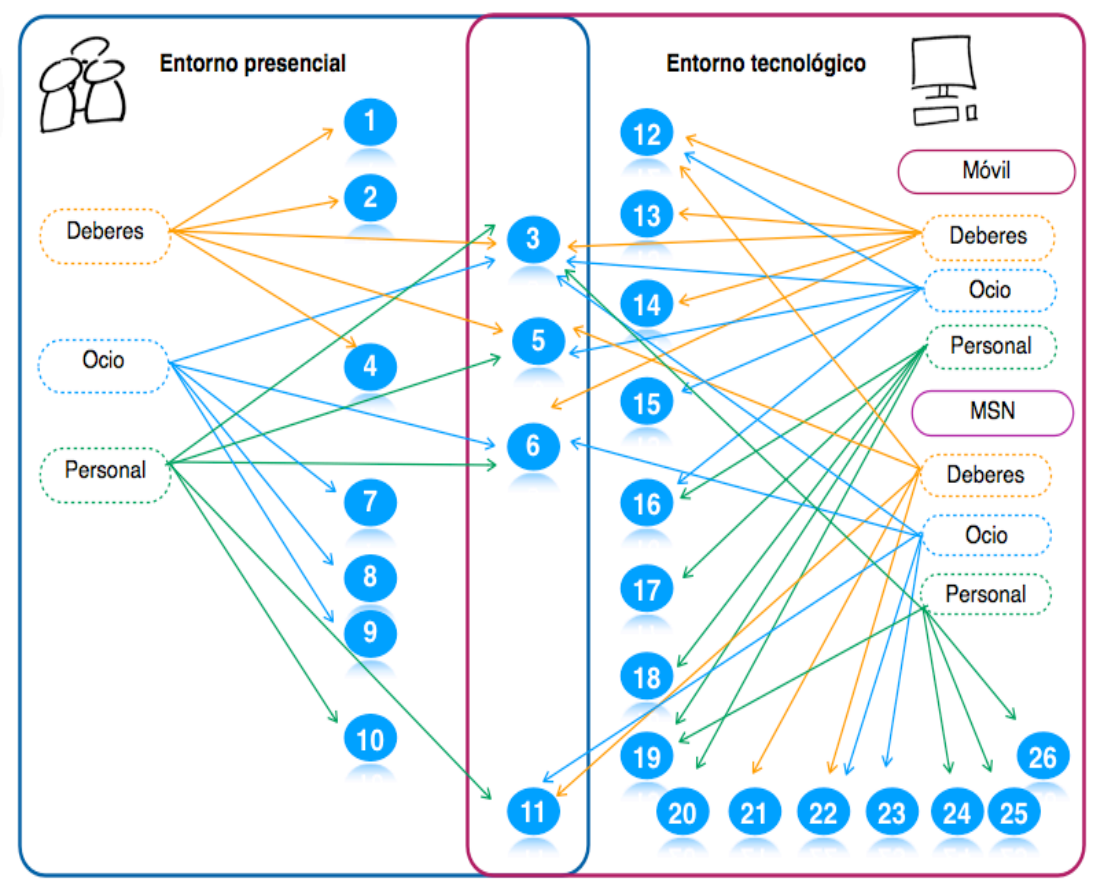

Figura 6. Ejemplo de mapa de interacción del "modelo tecnológico". 


\section{d) Modelo multirrelacional.}

Finalmente, el cuarto agrupamiento de alumnos (figura 6) se puede interpretar como una evolución de modelo 2 , que se ha separado porque en este caso los alumnos incluyen muchos contactos, más de 15 . Se caracteriza porque el número de relaciones que establece gracias a las redes es más numerosa y suele utilizar más herramientas que los demás grupos. Además, en este caso no es tan fácil identificar un entorno presencial y un entorno tecnológico diferenciado, porque las relaciones se entremezclan y crecen gracias a la red.

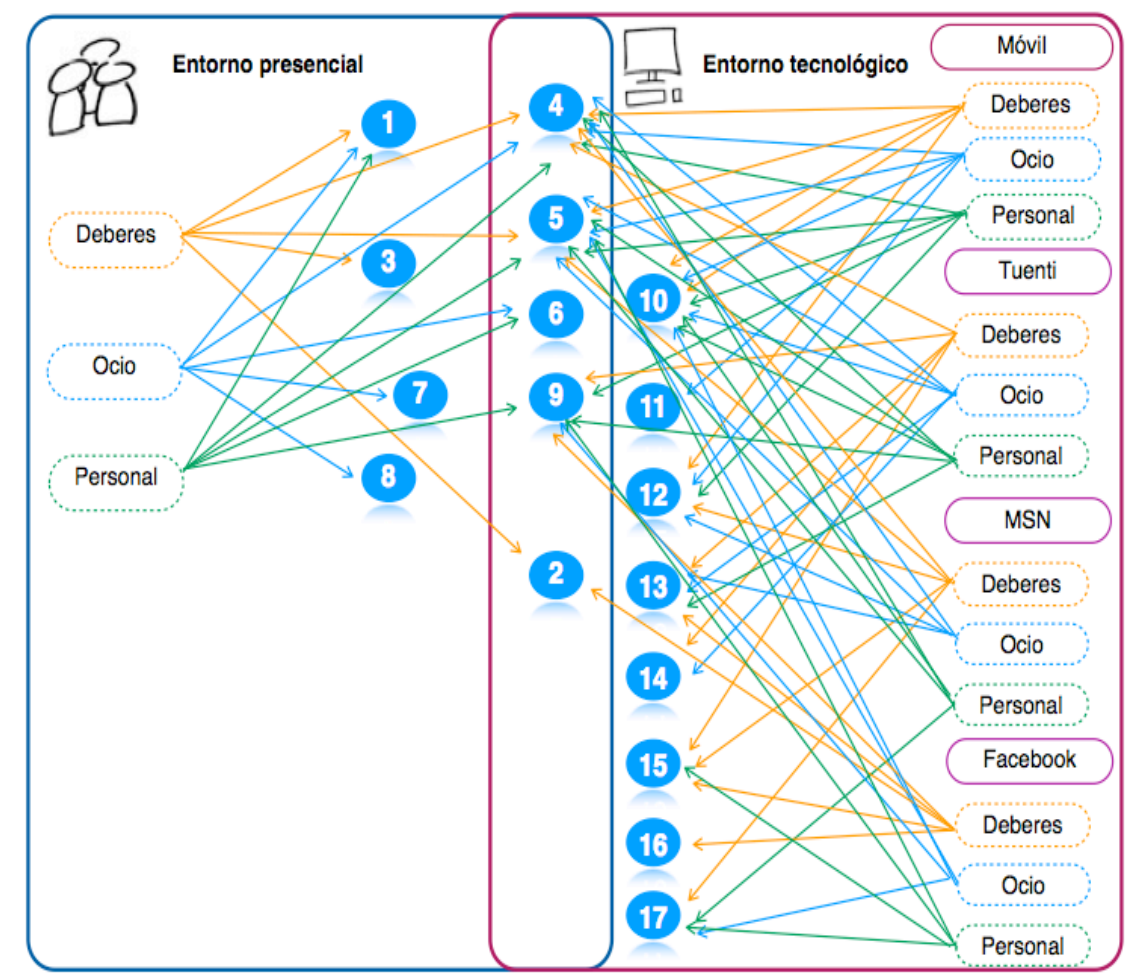

Figura 7. Ejemplo de mapa de interacción del "modelo multirrelacional".

\section{DISCUSIÓN DE LOS RESULTADOS}

Una de las conclusiones destacables del trabajo realizado se refiere al hecho de que entre los 14 y los 16 años el 53,2\% de los alumnos menciona contactos nuevos con los que se relaciona principamente en la red, de modo que el medio tecnológico sirve como mecanismo de socialización y apoyo de estas amistades, ya que estos contactos amplían los que tienen en su vida real y a menudo forman parte de su actividad cotidiana.

Por otra parte en un porcentaje significativo de los alumnos $(27,11 \%)$ se observa que los amigos presenciales generalmente son los mismos que se indican como amigos en entornos tecnológicos. Internet se concibe como una herramienta más para comunicarse con personas de su entorno, principalmente para realizar actividades relacionadas con el ocio.

Es igualmente destacable el caso del cuarto agrupamiento, ya que en el resto de grupos la herramienta messenger es identificada como la más utilizada, mientras que en este grupo de estudiantes es Tuenti la herramienta que tiene mayor uso, lo cual es razonable si pensamos en las potencialidades de comunicación de cada una. Messenger nos permite hablar con contactos que agregamos porque conocemos, sin embargo Tuenti nos ofrece continuamente sugerencias de nuevos contactos (amigos de amigos, personas con intereses similares que 
forman parte de la red, etc...) a incluir en nuestro listado de amigos, por tanto, Tuenti es precisamente la herramienta que permite al alumno crear ese amplio número de contactos que únicamente conoce de Internet. De hecho, los sujetos que más contactos tienen en el Messenger son los que se sitúan en el grupo 4, ya que un 50\% tiene más de 200 contactos. Por el contrario, partiendo del mismo número de contactos, los sujetos del modelo 1 "de costumbres" son los que menos contactos tienen $(27,1 \%)$.

Paradójicamente, los resultados referidos a la práctica de algún deporte (en la encuesta que se realizó) puestos en relación con estos sociogramas arrojan resultados diversos, ya que aunque si bien es cierto que los sujetos que no practican nunca deporte o lo hacen muy poco es similar en todos los grupos (aproximadamente un 30\%), los sujetos de los grupos 2 y 3 participan más a menudo en actividades deportivas (79,2\% y $76,2 \%$, respectivamente), que los sujetos del grupo 1 y el grupo 4 (53\% y 40,8\%, respectivamente)

En cuanto a la pertenencia del alumnado a grupos sociales o cívicos también apreciamos diferencias significativas, ya que los sujetos ubicados en el modelo 1 son los que menos participan en este tipo de actividades $(69,4 \%$ ) y los sujetos del modelo "multirelacional" los que más participan, con un porcentaje del $83,3 \%$. Por tanto, se comprueba que los sujetos que más interactúan en la red son también los que más lo hacen en un contexto presencial.

Podemos concluir que los sujetos que más utilizan Internet son los interactúan de acuerdo con las características del grupo 2 y el grupo 4, ya que el $75 \%$ en el modelo 2 y $80,9 \%$ en el modelo 4 utilizan Internet más de 11 horas a la semana. También se encuentra en el grupo 2 y 4 un mayor uso de las redes sociales.

Para finalizar, creemos que este estudio no sólo nos aporta una fotografía de cómo los adolescentes usan las herramientas tecnológicas para interactuar con sus iguales, sino que además puede ser de gran utilidad para definir acciones específicas de intervención educativa con ellos y puede igualmente servir como punto de arranque de nuestra reflexión sobre el desaprovechamiento educativo que estamos haciendo de las herramientas que los adolescentes tienen plenamente incorporadas a sus vidas.

\section{REFERENCIAS}

ARANDA, D., SÁNCHEZ, J., TABERNERO, C. y TUBELLA, I. (2010). Los jóvenes del siglo XXI: Prácticas comunicativas y consumo cultural. II Congreso Internacional AE-IC Málaga 2010. Comunicación y desarrollo en la era digital.

BOASE, J., HORRIGAN, J., WELLMAN, B., RAINIE, L. (2006). The Strength of Internet ties. Pew Internet \& American life project.

CASTELLS, M. (1996). La sociedad red. La era de la información: Economía, sociedad y cultura. Vol. 1. Madrid: Alianza Editorial.

CASTELLS, M. (2001). La galaxia Internet. Barcelona: Areté.

CASTAÑO, C. (2007). Herramientas telemáticas de apoyo a la Teleenseñanza. En J. Cabero Almenara, F. Martínez Sánchez y M.P. Prendes Espinosa (Coords.). Profesor, ¿estamos en el ciberespacio? (pp. 235-253). Barcelona: Davinci 
DOMíNGUEZ, G. y LLORENTE, M.C. (2009). La Educación social y la Web 2.0: nuevos espacios de innovación e interacción social en el espacio europeo de educación superior. Pixel-Bit. Revista de Medios y Educación, 35, 105-114.

DEL RíO, P. SÁBADA, F y BRINGUÉ, M. (2010). Menores y redes sociales: de la amistad al cyberbullying. Revista Estudios de la Juventud, 3.

EDEL, R. (2006). ¿Es usted padre de la genereación NET? Redcientifica. http://www.redcientifica.com/doc/doc200405149001.html [Consultado 14 de Noviembre de 2010]

HERRERO, J., MENESES, J., VALIENTE, L., RODRÍGUEZ, F. (2004). Participación social en contextos virtuales. Psicothema, 16 (3), pp. 456-460. Universidad de Oviedo y Universidad Oberta de Catalunya.

JACOBSON, A. (2001). Social Interactions and learning in an informal setting: an ethnography of communication in a knitting circle. Indiana University, US DEpartment of educatiob. Educational Resources Information. MF01/Pc01 Plus Postage.

KRAUT, R., PATTERSON, M., LUNDMARK, V., KIESLER, S., MUKOPHADHAYAY, T., SCHERLIS, W. (1998). Internet paradox: a social technology that reduces social involvement and psychological well-being? American Psychological Association. http://www.cs.cmu.edu/ kraut/RKraut.site.files/articles/kraut98-InternetParadox.pdf [Consultado el 1 de Marzo de 2011]

KRAUT,R., KIESLER,S., BONEVA,B., CUMMING,J. y HELGESON, V. (2001). Internet paradox revisited. Journal of Social Issues. 58, pp 49-74

KRAUT, R., FISH, R., ROOT, R., CHALFONTE, B. (2002). Informal Comunication in Organizations: forma, function, and technology. En IS. Oskamp \& Spacapan (eds). Human Reactions to Technology: the claremont symposium on applies social psychology. Beverly Hills, CA: Sage Publications.

MARTíNEZ, F. (2007). Implicaciones de la confusión. Conexión y comunicación ante las nuevas tecnologías de la información en la enseñanza. En J. Cabero Almenara, F. Martínez Sánchez y M.P. Prendes Espinosa (Coords.). Profesor, ¿'estamos en el ciberespacio? (pp. $37-$ 48). Barcelona: Davinci.

MARTÍNEZ, F. y SOLANO, I. (2003). El proceso comunicativo en situaciones virtuales. En F. Martínez Sánchez, (Coord.). Redes de comunicación en la enseñanza (13-30). Barcelona: Paidós.

MATUTE, H. (2002). ¿Es verdad que Internet produce depresión y aislamiento. 1er Congreso on-line Observatorio para la Cibersociedad. http://cibersociedad.rediris.es/congreso/comms/g21matute.htm [Consultado el 14 de Noviembre de 2010]. 
MENESES, G. (2006). Universidad: NTIC, interacción y aprendizaje. Edutec. Revista Electrónica de Tecnología Educativa, 20, 1-15.

MIR, B. (2010). La mirada pedagógica: ni nativos, ni residetentes... simplemente competentes digitales. En http://lamiradapedagogica.blogspot.com/2008/11/ni-nativos-niresidentes-simplemente.html [Consultado el 16 de Noviembre de 2011].

ORJUELA, L., CABELLO, P. y FERNÁNDEZ, I. (2010). La tecnología en la preadolescencia y adolescencia: usos, riesgos y propuestas desde los y las protagonistas. Un estudio de Save the Children. http://www.deaquinopasas.org/estudio_riesgos_internet.pdf [Consultado el 16 de Noviembre de 2010].

PRENDES, M. P. (2005). Los nuevos medios de comunicación y el aprendizaje en colaboración. Aula Abierta, 84, 127-146.

PRENSKY, M. (2001). Digital natives, digital inmigrants. On the Horizon. MCB University Press, $9 \quad$ (5). $\quad$ http://www.marcprensky.com/writing/prensky\%20\%20digital\%20natives,\%20digital\%20immigrants\%20-\%20part1.pdf [Consultado el 17 de Noviembre de 2010]

RED.ES (2005). Niños y jóvenes arrastran a los adultos al uso de tecnologías de la información en el hogar. Red.es: Sala de prensa.

http://www.red.es/prensa/notas/junio_05/05_06_27_tic.html [Consultado el 15 de Noviembre de 2010]

RIZO, M, (2006). Interacción y comunicación. Exploración teórico-conceptual del concepto de Interacción. En http://www.monografias.com/trabajos901/interaccion-comunicacionexploracion-teorica-conceptual/interaccion-comunicacion-exploracion-teorica-

conceptual.shtml [Consultado el 20 de Noviembre de 2010]

RODRIGUEZ, G., GIL, J. y GARCIA, E. (1996). Metodología de la investigación cualitativa. Málaga: Aljibe

WELLMAN, B. (2001). Physical place and cyberplace: changing portals and the rise of networked individualsim. Int. J. Urban Regional Res. Forthcoming

WHITE, D. (2010). Not natives and inmigrants but visitors and residents. En http://tallblog.conted.ox.ac.uk/index.php/2008/07/23/not-natives-immigrants-but-visitorsresidents/ [Consultado el 17 de Noviembre de 2010]

\section{Para citar este artículo:}

PRENDES, M. P.; SÁNCHEZ, M. M.; SERRANO, J. L. (2011) «Modelos de interacción de los adolescentes en contextos presenciales y virtuales» [artículo en línea]. EDUTEC, Revista Electrónica de Tecnología Educativa. Núm. 35 / Marzo 2011. [Fecha de consulta: dd/mm/aa]. http://edutec.rediris.es/revelec2/revelec35/

ISSN 1135-9250. 